\title{
An Intelligent Cross-Layer QoS-Aware Protocol with Traffic-Differentiation-Based for Energy Efficient Communication in WSNs
}

\author{
Jawad Ahmad Haqbeen ${ }^{1}$, Takayuki Ito ${ }^{1}$, Mohammad Arifuzzaman², Takanobu Otsuka' \\ ${ }^{1}$ Department of Computer Science \& Engineering, Nagoya Institute of Technology, \\ Nagoya, Aichi 466-8555, Japan \\ E-mail:jawad.haqbeen@itolab.nitech.ac.jp; ito.takayuki@nitech.ac.jp ; Otsuka.takanobu@nitech.ac.jp
}

\author{
Department of Electronics \& Communications Engineering, East West University, \\ Aftabnagar, Dhaka 1212, Bangladesh \\ E-mail: mazaman@ewbd.edu
}

\begin{abstract}
Despite an increase in energy efficiency of WSNs research in couple of last years, still the key challenge in the design of WSNs remains to maximize the quality of service (QoS) and energy efficiency of network system. The layered strength and functionality collaboration is commonly considered as one of the most promising techniques for dealing with the given challenge to increase the duration between recharging battery and improve energy efficiency in order to prolong the sensor nodes and network lifetime. This paper presents a novel Intelligent Cross Layer QoS-aware Protocol (I-XLP) with traffic differentiation for WSNs for monitoring applications which has been done by addressing cross layered, cluster-less and application specific design. The target application is building monitoring. The novel concept behind this paper is that, by leveraging classical layered approach and blending different layer functions our proposed protocol I-XLP achieves a solution for energy efficiency and latency for the case of building monitoring applications of WSNs. This is done by combining the strong functionalities of Medium Access control (MAC), Routing and Congestion control. The proposed protocol is energy efficient and the protocol ensures lower latency for the prioritized traffic. Our proposed I-XLP is simulated by using the network simulator (ns-2). The simulation results corroborate the theoretical idea, and verify that the proposed I-XLP transmits more data with less delay and its energy consumption compared with existing protocol achieves optimum energy efficiency.
\end{abstract}

Keywords: Wireless Sensor Networks (WSNs), Cross Layer Design, Quality of Service (QoS), Energy Efficiency.

\section{Introduction}

Wireless sensor networks (WSNs) is evolving areas of research due to its wide range of application in various domains, including health-care, assisted and enhancedliving scenarios, industrial and production monitoring, control networks, and many other fields [1]. In most of its applications, the WSN consists of a large number of wireless sensor nodes that are deployed randomly. The sensor nodes are typically small, and equipped with low-powered battery. Unlike other wireless networks, it is generally impractical to charge or replace the exhausted battery. Since prolonging lifetime of the sensor nodes is very important, energy efficiency becomes the most important attribute of design of Configuration of Page Layout communication protocol of sensor networks. Other attributes are fairness, latency, delivery ratio, and bandwidth [2]. During the last decade, a significant number of research works have been carried out on designing communication protocol for WSNs where particular interest is given on the performance metric of energy efficiency. Most of the works address the issue of energy efficiency by designing protocol for a particular layer of the classical 
layered architecture. For example in [3], [4] authors propose energy efficient MAC layer protocols. Similarly, [5], [6] concentrated on routing protocol design. From the state of art of the protocol designs for WSNs under the layered architecture, it is clear that the arena of research is in the state of maturity. However, the layer architecture has limitations, which do not rightly match with the physical architecture (resource constrained) of the sensor networks. On the other hand, cross-layer protocol design is actually desirable to achieve.

The greater performance for WSN is still far behind the state of maturity. It is important to acknowledge that during designing a protocol for a particular layer (i.,e., Transport, Network, MAC, or Physical layer) researcher and designer's objective is to maximize the performance in terms of the metrics related to that particular layer, which do not necessarily ensures the optimization of the overall network performance. On the other hand by violating the layered architecture optimum performance can be achieved. So by realizing the nature and objective of sensor network within the limitation of resources we advocate cross layer adaptation in the protocol design of WSN. Overall network performance gain can be achieved by the concept of cross layer approach which avoids the extra overhead as well as exploits actively the dependence between the protocols layers $[15,20]$. Moreover, in the traditional layered architecture there is a little scope of finding the tuneable solutions.

Our proposed I-XLP protocol is a cluster-less designed protocol. Cluster-based protocols are based on hierarchical network organization. Sensor nodes are grouped into clusters, with a cluster head elected for each cluster. Cluster members transmit their sensed or aggregated or relayed data to the cluster head. Cluster head takes the responsibility to communicate with the sink node. Using clusters has the benefit of limiting the area for flooding data to the cluster instead of the whole network, with positive consequences over scalability, lifetime and energy efficiency $[21,30]$.

Moreover, because nodes physically close usually sense similar events, data can be efficiently aggregated at the cluster head to obtain more precise data with avoiding redundancy. On the other hand, the benefits of the cluster-based solutions must be balanced against the signalling cost for cluster formation, cluster head selection and cluster maintenance [3]. Clustering is based entirely on smooth coordination between nodes. Since sensor nodes are interconnected by lossy links, ensuring a consistent state is complex. State inconsistency and race conditions can cause network instabilities, turning real-world deployments into a very challenging task. For these reasons, no clustering protocol has been standardized or used in commercial WSN products [3]. Furthermore, works such as [25] conclude that clustering doesn't increase the throughput of the network if all the nodes are homogeneous. And the work [26] proves that clustered networks do not necessarily outperform non-clustered WSNs.

It is known that, considering the most promising and practical applications of WSN, IETF ROLL identified the four potential applications of WSN namely industrial, urban, building, and home applications [1]. However, because of the wide range of application targeted, these requirements sometimes contradict each other, e.g. data delivery reliability is more important than energy efficiency in a refinery monitoring WSN, while it is the opposite in an urban -wide air quality monitoring WSN. So, it is evident that any particular protocol can't serve the best for applications of different (sometimes opposite) requirements. Besides, due to the wide range of WSN applications, without application specific design the quality of services cannot be easily ensured. Again, the nature of the most of the applications of WSN is such that only the sink nodes need to communicate with the outside world and the remaining nodes perform merely two duties; sensing data and forwarding packets of the neighbour towards the sink. So, these natures of sensor nodes which are quite different from the other kind of network can be exploited to achieve optimum network gain. Ideally, the deployed sensor network must be able to perform the best for achieving the objective for which it has been deployed. Considering the above discussion, it is clear that application specific protocol which is optimized for that particular application of WSN can be a good design principle rather than the protocol targeting to cover a wide range of applications.

Before initiating the design of communication protocol of WSNs, it is important to consider the accurate and thorough energy model of the different components of the targeted hardware. These components include the start-up energy for the transceiver, the static (distanceindependent) power drawn by the transmitter and receiver, power amplifier inefficiencies, coding energy, and protocol over-head [27]. For example, start-up energy for the transceiver must be considered before formulating sleep scheduling algorithm for sensor nodes. For sensor networks that transmit with short packet sizes occasionally, if static broadcast scheduling algorithm is used, even the start-up energy will defeat the energy consumption for transmission.

Transmission power adjustment is a very good technique for minimizing energy consumption of sensor nodes. This idea is based on power control protocol for wireless ad-hoc network $[5,25,26,27,29]$. A mechanism in order to improve the synchronization accuracy and scalability of slow flooding through deploying a clock speed agreement algorithm among 
sensor nodes helped many communication protocols in WSN to synchronize their notion of time [23]. In the literature, we find that usually there are four ways to use the transmission power for efficient communication. The first solution is to use a single transmission power in the whole network to reduce energy consumption which is referred as network level solution in [19]. The other options can be using a single transmission power for all neighbors, using different transmission power for different neighbors and the other option is using different transmission power for different packet. Though it is true that by using different transmission power level on the basis of the dynamics of the link quality is apparently the best option for energy savings but for making a good tradeoff between performance metrics as well as with consideration to computational complexity and additional overhead, the fixed transmission power for pairwise neighbors is the optimum design choice.

Furthermore, works such as [24] conclude that routing protocol with sink mobility can be achieved through direct forward sending approach, in this work data are sent straight to the sink node in direct approach in order to achieve energy efficiency for WSNs.

In this paper, we propose a novel Intelligent crosslayer QoS-aware protocol with traffic-differentiationbased for WSNs, where we tried to address cross layered, cluster-less and application specific design. The rest of the paper is organized as follows: In Section 2 concentrates on some related works. We define the system scenario and network model that we consider for our protocol design in section 3. In Section 4, we briefly describe the protocol stack of our proposed protocol, IXLP. We defined our protocol design principle and functionalities in section 5. In Section 6, we analyze the mathematical parameters of our proposed protocol. We evaluate and compared the performance of I-XLP with other combined protocols and discuss the simulation results in section 7. Finally, Section 8 concludes the works in the paper.

\section{Related Works}

In [7], an integrated MAC/routing protocol for geographical forwarding in WSNs is proposed. A competition is triggered at each hop in such a way that the most energy efficient transmission link is taken. Thus, packet progress towards the destination per transmitted power is taken into account to select the next hop. Without exchanging of location information, the protocol ensures energy efficiency and boosts data aggregation. In [8], authors propose a cross-layer design and optimization framework to improve the performance in WSNs. Authors use the concept of using an optimization agent to provide the exchange and control of information between the various protocol layers.

In [9], a novel cross-layer protocol "Breath" is proposed for control applications using WSNs where nodes, assumed to be attached to plants, must transmit information via multi-hop routing to the sink. Breath ensures a desired packet delivery and delay probabilities while minimizing the energy consumption of the network. The protocol is based on randomized routing, MAC, and duty-cycling jointly optimized for energy efficiency. In[10], a cross layer protocol (XLP) is introduced. The protocol includes congestion control, routing, and MAC functionalities in a cross-layer fashion. The design principle of XLP is based on initiative determination, which enables receiver-based contention, initiative-based forwarding, local congestion control, and distributed duty cycle operation in WSNs. In [11], authors present a physical (PHY) layer-aided MAC framework to enhance the uplink throughput of sensor data traffic. In their approach, the acknowledgements from the sensor nodes to the poll message are parallelized and by detecting the parallel acknowledgement, the allocation of channel resources is performed.

In [12], authors propose a solution to maximize the network utility. In this work, nodes autonomously determine their routing and transmission power in a dynamic environment. Authors also formulate the problem as a Markov Decision Process, and propose a distributed computation of the optimal policy. In [13], authors propose a novel cross-layer admission control mechanism to enhance the network performance and increase energy efficiency of a WSN, by avoiding the transmission of potentially useless packets. The protocol enhances the network performance by increasing the useful packet delivery ratio in high network loads. Also the protocol improves the energy efficiency at all loading condition. In [14], authors introduce a crosslayer operation model that improves the energy consumption and system throughput of IEEE 802.15.4 mobile WSNs by integrating four layers (application, routing, MAC and PHY). In [22], authors discussed different approaches of performance measurement of data aggregation techniques in WSNs, and attributes such as network life time, data latency and energy consumption surveyed. In [23], authors proposed a mechanism in order to improve the synchronization accuracy and scalability of slow flooding through deploying a clock speed agreement algorithm among sensor nodes. 
Our proposed I-XLP also includes congestion control, routing, and MAC functionalities in a cross-layer fashion. But I-XLP is different from XLP [10] in the sense that design principle of XLP is based on initiative determination, which enables receiver-based contention, initiative-based forwarding, local congestion control, and distributed duty cycle operation in WSNs. In our case, we try to address cross layered, cluster-less and application specific design with traffic differentiation based on quality of service.

\section{System Scenario and Network Model}

Building monitoring is chosen as the target application to apply the proposed protocol. However, it can be applied to any WSNs that have considerations for similar activities with similar performance metrics requirements.

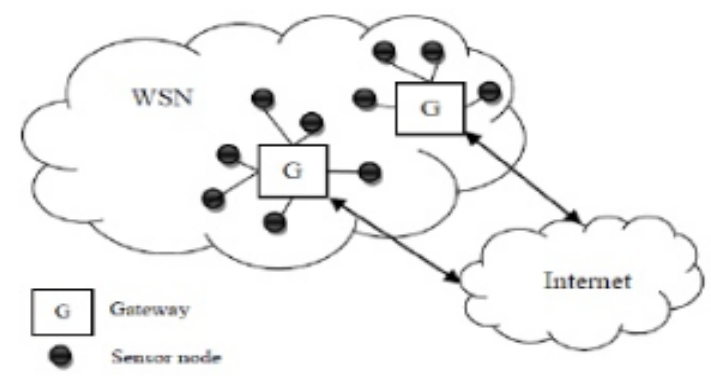

Fig. 1 High level view of building automation by WSN integrated with Internet.

We consider a building equipped with a WSN where sensor nodes monitor the power consumption of the appliance in the building. The nodes will perform the duty of smoke detector within building as well, and report alarms to fire monitoring hubs, i.e. gateway in figure 1.

Sub-headings should be typeset in boldface italic and capitalize the first letter of the first word only.

Typeset sub-subheadings in medium face italic and capitalize the first letter of the first word only.

The network is modeled by a Directed Acyclic Graph (DAG), $G=(V, L)$ where $V$ is the set of sensor nodes and $L$ is the set of directed links. Each link $l$ is from a transmitter node to a receiver node (towards the destination node). The necessary condition for a link exists from node $i$ to node $j$ is that, if the received power

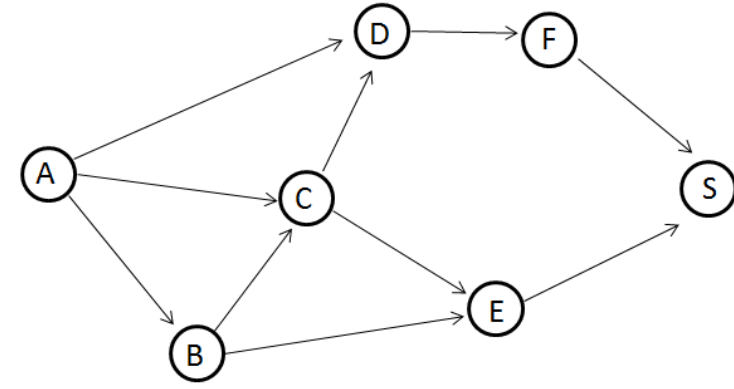

Fig. 2 Assumed Network model for I-XLP, directed Acyclic graph.

at node $j$, when node $i$ transmits at maximum transmission power and no interference is present, is greater than a predefined threshold.

\section{Protocol Stack of I-XLP}

The terminology that has been used in I-XLP are slot, duty cycle and rendezvous slot. A slot has been defined in our protocol as a periodic interval and consists of two periods, active and sleep periods. We call duty cycle as a proportion of active period part to entire cycle time. Finally, A rendezvous slot in our paper is define as a slot which is explicitly dedicated to a pair of sensor nodes for each other communication, in order to communicate with each other.

I-XLP classifies packets according to their importance (i.e. delay requirements) and stores the packets into the appropriate queue. The source node knows the degree of importance of the sensed data and accordingly the application layer sets the priority. Application layer does it by appending 1 extra bit at the end of the data packet. Figure 3 shows the format of each data packet.

\begin{tabular}{|c|c|c|}
\hline Header & Data & $\begin{array}{c}\text { Extra 1 bit } \\
\text { (used for priority) }\end{array}$ \\
\hline
\end{tabular}

Fig. 3 Format of each data packet for proposed protocol I-XLP

The mechanism of I-XLP is based on dividing the communication time into fixed length slots or frames. The contents of each slots are shown in Figure 4. Each slot begins with a SYNC period. The purpose of the SYNC packet is to maintain synchronization between the nodes within the same virtual cluster. The next part of the active period of the frame is reservation slot which is used for the data slot reservation. Finally, the last part is used for data and ACK transmission by sensor nodes. Generally, each slot in I-XLP is a periodic 
interval which combines from three periods, first of all fixed length SYNC period, second one is fixed length listen period which use for Request-To-Send (RTS) and Clear-To-Send (CTS). The third number period which our protocol slot contains is sleep period. All the three periods of slot depend on the duty cycle.

\begin{tabular}{|l|c|c|c|}
\hline \multicolumn{2}{|c|}{ Active Period } & \multicolumn{3}{|c|}{ Sleep Period } \\
\hline \multirow{2}{*}{$\begin{array}{c}\text { RTS/ } \\
\text { CTS }\end{array}$} & Data & Ack \\
\hline Random Access: & Scheduled Access
\end{tabular}

Fig. 4 Contents of a slot or frame for the proposed I-XLP protocol.

\subsection{Clustering and Synchronization of I-XLP}

Frame synchronization in I-XLP is done by virtual clustering, as described in [3]. The time when node comes to life, it starts by waiting and listening. So if the node hears nothing for a certain period, it chooses a frame schedule and transmits a SYNC packet. The packet of SYNC contains the time until the next frame starts. If the node during start up hears a SYNC packet from another node, it follows the schedule in that SYNC packet and transmits its own SYNC accordingly. Nodes retransmit their SYNC once in a while. When a node has a schedule but it hears SYNC with a different schedule from another node, then it adopts both schedules. And here adopting both schedules ensures the successful communication among the nodes of different schedule. The described synchronization scheme, which is called virtual clustering [3], urges nodes to form clusters with the same

schedule. So by this procedure, all the nodes in the networks need not to follow the same schedule.

During virtual cluster creation of I-XLP, each node creates the one hop neighbor list and with using these a node can easily constitute the two hop neighbor list. After that each node is given an ID number, such that within a two hop neighbor the ID is unique.

\subsection{Slot Assignment of I-XLP}

In this sub-section the slot assignment of I-XLP is briefly described.

Each slot in our proposed protocol consists of a fixed length SYNC period, a fixed length RTS/CTS period and a Sleep period including data that depends on the duty cycle as shown in Figure 4. The duty cycle should be chosen in such a way that the sleep period of a slot is large enough to transmit a data packet along with ACK. All nodes have the permission to transmit in any slot but the node that has a critical data (with high priority) will get the priority. The priority in a slot can be ensured by choosing contention window size.

Contention window size is used to set critical (data with high priority) or not critical (data with no priority). The critical data set by using different contention window sizes for data type of priority and non priority. Critical data of a slot picks a random time uniformly over contention interval [1, (CW)CTL], while on another side non critical data do so within [1, (CW) NCTL]. The average window size observed by critical data node would be $[1+(\mathrm{CW}) \mathrm{CTL}] / 2$ and for the non critical data would be $[(\mathrm{CW}) \mathrm{CTL}+1+(\mathrm{CW}) \mathrm{CTL}] / 2$. The critical data takes hold of the channel every time because of its smaller contention window, provided that critical data of slot has some data to send. Once the slot has been chosen by data, the node transmits at that slot. So in this case for both the critical (with high priority) and non critical (with no priority), SYNC and RTS transmission in our proposed protocol starts by waiting and listening for a random time within the contention interval.

So, I-XLP ensures higher priority for critical traffic. The protocol does it by using smaller contention window size for critical traffic. When a node acquires a data to transmit, it first checks whether the data is a critical data or not. If it is a critical data, the node takes a random back-off within affixed time period, $\mathrm{T}_{\mathrm{c}}$. When the backoff timer expires, the node runs Clear Channel Assessment (CCA) and if the channel is found clear, the node transmits data. If the channel is busy then it waits until the channel becomes free and repeats the above process. Now, if the data is not a critical data then the node waits for $\mathrm{T}_{\mathrm{c}}$ and then performs a random back-off within a contention window $\left[\mathrm{T}_{\mathrm{c}}, \mathrm{T}_{\mathrm{o}}\right]$. When the back-off timer expires, the node runs Clear Channel Assessment (CCA) and if the channel is clear it starts transmission. If the channel is busy then the node waits until the channel becomes clear and repeats the above process. It should be noted here that when a slot is already declared as rendezvous slot for that slot without waiting for contention window the node can initiate transmission same as a Time Division Multiple Access (TDMA) link scheduling.

\begin{tabular}{|l|l|l|l|l|l|l|l|l|l|}
\hline $\mathrm{N}_{1}$ & $\mathrm{~N}_{2}$ & $\mathrm{~N}_{3}$ & $\mathrm{~N}_{4}$ & $\mathrm{~N}_{5}$ & $\mathrm{~N}_{6}$ & $\mathrm{~N}_{7}$ & $\mathrm{~N}_{8}$ & $\mathrm{~N}_{1}$ & $\mathrm{~N}_{2}$ \\
\hline $\mathrm{S}_{1}$ & $\mathrm{~S}_{2}$ & $\mathrm{~S}_{3}$ & $\mathrm{~S}_{4}$ & $\mathrm{~S}_{5}$ & $\mathrm{~S}_{6}$ & $\mathrm{~S}_{7}$ & $\mathrm{~S}_{8}$ & $\mathrm{~S}_{9}$ & $\mathrm{~S}_{10}$ \\
\hline
\end{tabular}

Fig. 5 Critical data selection of each frame for 8 node using module 8 
The critical data calculation in I-XLP can be preformed by each node locally by using simple clock arithmetic. In fig. 5 , there are 8 neighbor nodes where every node at least has 1 or 2 hop neighbor to one another. The nodes $\mathrm{N} 1$ will be the critical data of the slot S1 and S2, where $\mathrm{N}$ represent the sensor node and $\mathrm{S}$ represent the slot sequences.

And according in fig. 6, the sensor node N1 is the critical data of the slot S9 and S17.

\begin{tabular}{|l|l|l|l|l|l|l|l|l|l|}
\hline $\mathrm{N}_{1}$ & $\mathrm{~N}_{2}$ & $\mathrm{~N}_{3}$ & $\mathrm{~N}_{4}$ & $\mathrm{~N}_{5}$ & $\mathrm{~N}_{6}$ & $\mathrm{~N}_{7}$ & $\mathrm{~N}_{8}$ & $\mathrm{~N}_{1}$ & $\mathrm{~N}_{2}$ \\
\hline $\mathrm{S}_{9}$ & $\mathrm{~S}_{10}$ & $\mathrm{~S}_{11}$ & $\mathrm{~S}_{12}$ & $\mathrm{~S}_{13}$ & $\mathrm{~S}_{14}$ & $\mathrm{~S}_{15}$ & $\mathrm{~S}_{16}$ & $\mathrm{~S}_{17}$ & $\mathrm{~S}_{18}$ \\
\hline
\end{tabular}

Fig. 6 Critical data selection of each frame using module 16

Now, each node can make some slot as a rendezvous slot with which it can send message to its neighbor exclusively. In I-XLP, both the critical data and rendezvous slots will be calculated by each node locally using clock arithmetic, as modulo $\mathrm{m}$. The value of $\mathrm{m}$ in module is set according to the system requirements, i.e. network load, delay, message buffer size etc. Here in module $(\mathrm{m}), \mathrm{m}$ will be always multiple of node ID. For example, let sensor node 2 want to create a rendezvous slot. By using module 8 , the rendezvous slots of node 2 will be a subset of $[2,10,18 \ldots]$ and by using module 16 the rendezvous slot for node 2 will be a subset of $[2,18 \ldots]$. It is explained in figure 6 where S9, S10.. represents the slot sequence and $\mathrm{N} 1, \mathrm{~N} 2 \ldots$ represents the sensor nodes. If we use modulo 8 , node $\mathrm{N} 2$ can make slot $\mathrm{S} 10$ as its rendezvous slot and if modulo 16 then it is S18. It is worth mentioned that, though node $\mathrm{N} 2$ is the critical of both slots S10 and S18 but sensor node N2 cannot make $\mathrm{S} 10$ as its rendezvous slot because $\mathrm{S} 10$ is not a subset of $[2,18 \ldots]$. For the scalability point of view, the value that we used it during modulo operation as $\mathrm{m}$ will be always larger than the number of 2-hop neighbor nodes in a virtual cluster because when if there is some new node that wants to join in the network, at least there will be some slots which are not using as rendezvous and it is used for the scalability. Figure 7 shows how I-XLP sensor node ensures energy saving and clarifies its timing scheme for energy saving and it shows that the energy saving scheme timing diagram for our proposed protocol.

\section{I-XLP Design Principle and Functionality}

In this section the design principle and cross layer functionalities (Link layer, MAC, Routing and congestion control) are briefly described sequentially for comprehensible readability. Cooperation, compromise, and group participation of different layer functionalities for achieving a unified goal are also briefly explained here.

We adapt a modified version of intelligent hybrid MAC (IH-MAC) [2] protocol for wireless sensor network, in our proposed I-XLP protocol, in order to achieve optimized performance with adapting the other protocol stack. Each node will sleep for some time and then periodically wakes up to see whether any other node wants to talk to it. During sleeping, the node turns off its radio, and sets a timer to awake later. If a node receives a data (either sensed data or data from the neighbor) it will first check the status of the data, whether it is high priority (or critical) data or not. Sensor nodes energy saving scheme timing diagram of I-XLP, are shown in figure 7 . The node which has any critical data (i.e. low latency requirement) gets the priority for transmission. If there is no such data, then the nodes intended to transmission in the neighborhood will contend fairly with each other in carrier sense multiple access (CSMA) manner. If there is more than one node having critical data, then the contention will take place between them. The priority service to the critical data is ensured in two manners. Firstly, the node having the critical data will use a smaller contention window size. Secondly, to increase the probability of successful transmission (avoid collision) of critical data, as soon as the neighbor nodes know the existence of the critical data, it will stop its transmission of noncritical (regular) data for the next cycle. Besides, since there exist several paths/links towards the destination (sink), the system in general will decrease the collision probability. In I-XLP, broadcast packets are sent without Request-To-Send (RTS) and Clear-To-Send (CTS). Unicast packets will follow the sequence of RTS, CTS, Data, and Acknowledgement (ACK). This scheme is well recognized and used, for example in the IEEE 802.11 standard [21]. 


\begin{tabular}{|c|c|c|c|c|c|c|c|c|}
\hline $\operatorname{Node}_{A}$ & $\begin{array}{l}Z \\
\underline{I} \\
\underline{\omega} \\
\underline{\Xi}\end{array}$ & 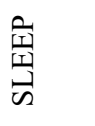 & $\begin{array}{l}Z \\
Z \\
\underline{\Xi} \\
\tilde{\Xi} \\
\Xi\end{array}$ & 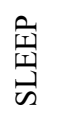 & 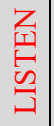 & DATA & 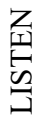 & 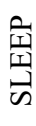 \\
\hline Node $_{B}$ & 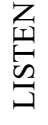 & DATA & 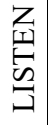 & $\begin{array}{c}\text { DAT } \\
\text { A }\end{array}$ & \multicolumn{2}{|c|}{ SLEEP } & 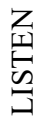 & 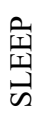 \\
\hline Node $_{C}$ & 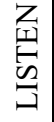 & DATA & 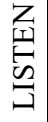 & $\begin{array}{l}\overrightarrow{|r|} \\
\overrightarrow{\mid r} \\
\vec{\sigma}\end{array}$ & $\begin{array}{l}z \\
\underline{y} \\
\omega \\
\Xi \\
\exists\end{array}$ & DATA & 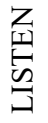 & $\begin{array}{l}\stackrel{\overrightarrow{I I}}{\underline{I}} \\
\vec{\Xi}\end{array}$ \\
\hline Node $_{\mathrm{D}}$ & 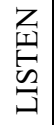 & 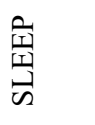 & \begin{tabular}{l}
$Z$ \\
\multicolumn{1}{|c}{} \\
$\underline{\tilde{\Omega}}$ \\
$\underline{\Xi}$
\end{tabular} & $\begin{array}{c}\text { DAT } \\
\text { A }\end{array}$ & & EEP & 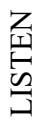 & 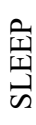 \\
\hline & Slo & & Slo & & Slot & & Slo & \\
\hline
\end{tabular}

Fig. 7 Sensor nodes energy saving scheme timing diagram of I-XLP (the red portion is rendezvous slot of our protocol and remaining slots are like other protocols)

I-XLP supports parallel transmission[15] in case of noncritical data. The couple of nodes are allowed to communicate simultaneously, can be found in the neighbors list table. So, by obeying the status of the mutually exclusiveness of the two links in the neighboring list, only those sensor nodes who have an intention to communicate will contend for successful agreement of data transfer. It should be mentioned that, since the RTS message contains the link information (both sender and the receiver) after seeing the first RTS, the number of candidate nodes for desiring the access to the medium will be considerably decreased to meet the parallel transmission requirements.

During the sleep state a node turn off its radio and start a timer whose duration is predefined according to the duty cycle of the protocol with consideration of the existence of rendezvous [16] communication between any pair of nodes. When the timer expires the node goes to wake-up state. It turns on its radio and switches to listen to the data channel and it goes to idle listening state. If the node has any data to send or receive it goes in the carrier sense multiple access with collision avoidance (CSMA/CA) state otherwise after time out it goes to sleep state. If the sender node wins the contention both the sender and the intended receiver go to the $\mathrm{Tx} / \mathrm{Rx}$ state and go to sleep state after successful communication. Nodes that fail contention go to sleep state.

We use in our work the concept of gradient to design the routing layer functionalities for our proposed I-XLP. The routes are organized along a Destination Oriented Directed Acyclic Graph (DODAG), a category of Directed Acyclic Graph. The destination node is node $\mathrm{S}$ (sink node) in the Figure 2. On each link the value of average (required energy and required time) matrices is collected. And by adding the values a node can know the approximate value of consumed energy and required time towards the sink node which is used for choosing the next hope neighbors. However, depending on the traffic scenario of a particular link, alternative neighbor can be chosen by the routing protocol and this load balancing is done with a cooperation of MAC protocol. Thus the routing of I-XLP provides paths from routers to a sink while requiring routers to store very little forwarding and routing table information, which is compatible with the resource constraint of wireless sensor nodes.

Now let us see from Fig. 2, how the source node (nodes other than S) can find the best next hop towards the sink node (node $\mathrm{S}$ ). For example, in figure 2 while node B and node E work on link scheduling [5], node A will be aware of it, in other words, node A cannot find node B when it wakeup and wants to transmit a packet to B. So, node A will automatically transmit a packet to $\mathrm{C}$ or $\mathrm{D}$ based on the suboptimal multi-hop path cost. It can be noted here that, the main objective of our proposed IXLP is to achieve an efficient solution for WSN application of building monitoring, however the protocol can be extended for achieving other performance objective by considering different metrics like bandwidth, packet delivery ratio etc. And addition, multiplication etc., technique can be used to combine the link cost in order to calculate multi-hop path cost.

Congestion control is also considered. More importantly it is automatic. The congestion is considered on the link by link basis and so there is no need for considering end to end congestion control. A packet can only be injected by a node if there is a good neighbor who is not performing rendezvous [5]. When a particular link is already loaded with heavy traffic (long queue) the node begins to perform in link scheduling (rendezvous) manner with an explicit declaration [5].

\subsection{I-XLP Data Transmission Feature}

Now let us consider Fig. 2 a DAG graph which our network modeled has 7 nodes A, B, C, D, E, and S. The transmission is within the range of each other nodes, in order to explain simple case scenario of I-XLP transmission mode and its energy saving scheme we consider fig. 7 , slots namely $\mathrm{i}_{0}, \mathrm{i}_{1}, \mathrm{i}_{2}$ and $\mathrm{i}_{3}$ 
consecutively. Each slots contains two portions, Listen and Sleep part. The Listen part of slot is used for Synchronization (SYNC), Request-To-Send (RTS) and Clear-To-Send (CTS). The Sleep portion of slot is mainly used for data transmission between sensor nodes. The proportion interval of these two part of slot depends to the operation of duty cycle. Now, we take some arbitrary transmission in order to clarify the working concept of I-XLP. Let consider that data transmission occurs between two nodes of network during slot $i_{0}$ namely $B$ and $C$. The same time the node $\mathrm{A}$ and node $\mathrm{D}$ woke up in order to see whether any data send to them or not. But at the same time node A and node D subsequently go back to sleep mode because they lose contention or there is no data for them to receive. The same case occurs in Slot $i_{1}$ where transmission occurred between two sensor node B and D. But difference happens when on the Slot $\mathrm{i}_{2}$ the sensor node $\mathrm{A}$ and node $\mathrm{C}$ create rendezvous between them. So, on that slot node B and node D will not wake up from their sleep, instead they continue to sleep for the whole life time of the slot. The node B and node D ensures energy efficiency by their zero duty cycle operation, sleep time lingering and avoiding data transition between two part of slot. The node A and node $\mathrm{C}$ will ensure saving energy by avoiding RTS, CTS and contention for getting the slot. Thus, we see by creating rendezvous slot the energy efficiency enhance for all nodes in two hop neighbor whether they participate in data transmission or they do not. Therefor, the adjusting data transmission of I-XLP helps the energy efficiency of power saving feature of the protocol.

\section{Mathematical Analysis}

The main objective of this section is to define the numerical constructive approach of our protocol during path selection procedure which is efficient for the terms energy efficiency and latency.

1) Shortest Constrained Path: The key philosophy of a protocol path selection is to support constraint-based routing where constraints may be applied to both link and nodes. To do so, I-XLP support constraint-based routing. If a link or a node does not satisfy a required constraint, it is "pruned" from the candidate neighbor set, thus leading to the shortest path [10].

2) Node constraint in I-XLP: We calculate the relay rate threshold [16] to find the availability to receive additional relay packet. The overall input packet at node $i, \lambda_{i}$ can be represented as

$$
\lambda_{i}=\lambda_{i i}+\lambda_{i, \text { relay }}=\lambda_{i i}+\sum_{j \in N_{i}^{i n}} \lambda_{j i} .
$$

Where $\lambda_{i i}$ is the generated packet rate, $\lambda_{j i}$ is the relay packet rate from node $\mathrm{j}$ to node i. $N_{i}^{i n}$ is the set of nodes from which node $i$ receives relay packets, and $\lambda_{i, r e l a y}$ is the overall relay packet rate of node $i$. Node $i$ aims to transmit all the packets in its buffer and thus, the overall output rate of node $i$ is given by

$$
\mu_{i}=\left(\lambda_{i i}+\lambda_{i, \text { relay }}\right)+e_{i}\left(\lambda_{i i}+\lambda_{i, \text { relay }}\right) .
$$

Where $e_{i}$ is the packet error rate, hence $e_{i}\left(\lambda_{i i}+\right.$ $\left.\lambda_{i, \text { relay }}\right)$ can be used to approximate the retransmission.

To, prevent backlog at a node, the generated and received packets should be transmitted during the active period of duty cycles of a node for a given time. If we consider a time span of $\mathrm{T}$ seconds because of duty cycling a node is active for the period of dT, where $d$ represents the duty cycle. Thus,

$$
d T \geq\left[\left(1+e_{i}\right) \lambda_{i i}+\left(2+e_{i}\right) \lambda_{i, \text { relay }}\right] T T_{a v g} .
$$

Where $T_{\text {avg }}$ is the average time needed to transmit the packet after reception. It includes the queuing time and MAC overhead.

Hence the availability of a node in the path is determined by

$$
\lambda_{i, \text { relay }} \leq \lambda_{i, \text { relay }}^{T h}
$$

where,

$$
\lambda_{i, \text { relay }}^{T h}=\frac{d}{\left(2+e_{i}\right) T_{a v g}}-\frac{\left(1+e_{i}\right)}{\left(2+e_{i}\right)^{\lambda_{i i}}} .
$$

3) Link constraint in I-XLP: Link constraint is defined as the node hasn't reached the threshold value of the incoming traffic (sum of generated and received), however it is performing link scheduling with a particular neighbor. For detail about link scheduling operation interested reader is encouraged to go through [4].

4) Shortest Energy Efficient path: Path offering the minimum end-to-end energy consumption.

5) Shortest delay path: Path offering the shortest endto-end delay. This delay is calculated at each hop and piggybacked to the source node through acknowledgement field for MAC frame. The Estimated time to destination (ETD) can be approximated by summing up the latency at each hop towards the path to the sink.

$$
\operatorname{ETD}(h)=\sum_{i=1}^{h}\left(q_{i}+\alpha_{i}+\beta_{i}\right)
$$


Where $\alpha_{i}$ is the delay generated due to the random back-off taken by the node before running Clear Channel Assessment (CCA), which can be characterized by back-off mechanism of carrier sense multiple access with collision avoidance (CSMA/CA) of IEEE 802.11 [23], $\beta_{i}$ is the delay generated if the node and its neighbor do not belong to the same virtual cluster and $q_{i}$, is queuing delay. Generally, in WSNs a larger queuing occurs in a node when it receives more packets than it can forward i.e., excessive packets received by a node eventually result in an excessive queue length. In I-XLP, the relay traffic admission to a node's queue is restricted after reaching to a threshold value. However, in case of lower packet inter-arrival rate (higher generated traffic) queue is still formed.

\subsection{I-XLP Path Selection Procedure}

In this sub-section, we explain how the MAC operations assist the Routing techniques of I-XLP in the selection of appropriate path (paths defined in section 4 and 5) based on the performance objective.

We use the concept of gradient to design the routing layer functionalities for our proposed I-XLP. The routes are organized along a Destination Oriented Directed Acyclic Graph (DODAG), a category of Directed Acyclic Graph [21, 22]. The destination node is node S (sink node) in figure 2 . On each link the value of average (required energy and required time) metrics are collected. Then by adding the values a node can know the approximate value of consumed energy and required time towards the sink node which is used for choosing the next hope neighbors. Besides, depending on the traffic scenario of a particular node or link, alternative neighbor can be chosen by the routing (i.e., Shortest Constrained path) and this load balancing is done with a cooperation of the information accumulated as a byproduct of MAC operation. Thus the routing of I-XLP provides paths from routers to a sink while requiring routers (nodes of sensor network) to store reduced forwarding and routing table information.

\section{Result and Discussion}

In this section, in order to measure the effectiveness and evaluate the performance scalability of the proposed IXLP, we took some classical layered protocol from their corresponding state of art works and make different combination of the protocols to investigate and compare the performance of our protocol. The performance metrics used in the performance evaluation of I-XLP protocol are energy consumption per bit, data delivery ratio and average packet latency. For routing layer, we consider geographic[17], for MAC layer we choose duty cycling MAC (S-MAC [3], T-MAC[4], and CSMA with duty cycle, and for transport layer we pick up ESRT[18] protocol. Since most of the applications of WSNs require location information, including our considered application which is building monitoring applications, there is no overhead to reuse this location information for communication purposes; that is reason behind the taking of geographical routing for comparisons. On the other hand, duty cycling MAC is obviously the precise selection for energy constraint wireless sensor node and for the case of ESRT (Event-To-Sink-Transport) protocol, it is the well-matched transport layer protocol for our considered application which can be implemented with the least complexity as well.

We simulate both cases of prioritized traffic and nonprioritized traffic. For non-prioritized traffic, all the generated traffic has the equal priority and in case of prioritized traffic we use the $5 \%$ of the total traffic with critical or high priority and it is generated randomly. In simulation, we compare the integrated performance of Congestion control, Routing and MAC layer of I-XLP with other three layered protocol which are configured as following combinations:

(a) Flooding + CSMA: For getting access to the medium, CSMA protocol is used by each sensor node. When a node broadcasts a packet, among the neighbor nodes (receiver nodes) only nodes who are closer towards the sink compared to the sender node, rebroadcast the packet in the similar fashion. This configuration is made by only network and MAC layer protocol and no transport layer protocol/congestion control is integrated.

(b) Greedy Perimeter Stateless routing (GPSR) + SMAC+ ESRT: In this configuration, for routing layer greedy perimeter stateless routing is taken and for medium access control, standard S-MAC is used. Congestion is controlled by the ESRT protocol.

(c) Greedy Perimeter Stateless Routing (GPSR) + TMAC+ ESRT: This configuration is similar with the previous configuration. However, instead of S-MAC, TMAC protocol is chosen for Medium access control and instead of greedy geographical routing, GPSR is chosen for routing layer. And for congestion control ESRT is chosen like the previous configuration.

\subsection{Simulation Setup and Environment}

The performance of the proposed I-XLP is evaluated using network simulator ns-2. In the simulation setup, we took 300 sensor nodes which deployed on an area grid of $100 \mathrm{~m} \times 100 \mathrm{~m}$. The nodes are static and are distributed in a uniformly random way on the grid. Each sensor has transmission range of $20 \mathrm{~m}$. The sink node is chosen on the bottom right corner of the grid. The size of a data packet is 30 bytes and the data transmission 
rate is set to $250 \mathrm{kbps}$. The generation interval varies from 1 to 10 seconds. The results are shown both for the prioritized traffic and non-prioritized traffic. The simulation parameters are listed in Table I.

Table I. Simulation Parameter Used For The I-Xlp

\begin{tabular}{|l|l|}
\hline Parameter Names & Value \\
\hline Transmission energy & $24.75 \mathrm{~mW}$ \\
\hline Receive energy & $13.5 \mathrm{~mW}$ \\
\hline Idle energy & $13.5 \mathrm{~mW}$ \\
\hline Sleep state & $15 \mu \mathrm{W}$ \\
\hline Channel Bandwidth & 30 bytes \\
\hline Data transmission & $250 \mathrm{kbps}$ \\
\hline Data Packet length & 30 bytes \\
\hline Frame length & 1 sec \\
\hline Data Packet length & 100 bytes \\
\hline Control Packet length & 20 bytes \\
\hline Packet generation Interval & $1-10 \mathrm{sec}$ \\
\hline Prioritized Traffic & $5 \%$ \\
\hline
\end{tabular}

\subsection{Simulation Results}

In this section, we discuss the comparative performance metrics used in I-XLP. The comparative performance of the studied and evaluated routing protocols is in terms of energy consumption per bit, latency and packet delivery ratio for the case of prioritized and non prioritized data traffic. The results of evaluation corresponding to the averaged energy consumption for non-prioritized traffic for different protocol are shown in figure 8 . We see that energy consumption increases with the increase of duty cycle for all four protocols; however, our proposed protocol clearly outperforms other three for all value of the duty cycle. Because in IXLP the dynamic gradient routing for routing functionalities combined with CSMA and TDMA (broadcast scheduling and link scheduling) in the MAC functionalities. Furthermore, the novel scheduling technique in the MAC layer of the I-XLP assists the routing layer to choose optimum route (next hop) for a packet. On the other hand, for using the dynamic gradient routing, the MAC layer can easily eliminate the bad neighbors (during scheduling) who are not able to forward the packet to the destination with a reasonable delay. We notice these collaborations between layers minimize the energy consumption and save significant amount of energy. Similarly, the strength of combined contention based and schedule based approach of MAC layer of the I-XLP assists to achieve significant amount of energy savings. In case of other three layered protocol, when routing layer chooses a neighbor node for transmission, because of the use of duty cycling, in most of the cases, MAC layer cannot immediately match the sender node and receiver node simultaneously and the delay ends with a result of higher energy consumption.

About the prioritized traffic, as figure 9 shows, we see that energy consumption per bit of I-XLP is not much effected with the introduction of differential traffic (traffic with different priority). It should be noted that when we use the term of non-prioritized traffic, it means that all the traffic has the equal priority. And in case of prioritized traffic we use (consider) the $5 \%$ of the total traffic with critical or high priority and it is generated randomly. We vary the packet generation interval, so we see that average energy consumption per bit of I-XLP is less than the consumption of other two protocols. It is because during prioritized traffic our protocol makes some rendezvous slot and as we defined that during rendezvous slot creating energy is less consumed.

In figure 10 we compare the latency of our proposed protocol with other three protocols in case of nonprioritized traffic. In terms of latency our proposed protocol performs similar to GPSR + S-MAC+ ESRT and outperforms the other two protocols.

Figure 11 shows the latency of our proposed protocol with other three protocols in case of prioritized traffic. We see that I-XLP serves the high priority traffic with the lowest minimum delay. However, low priority traffic experience a slightly higher delay compared to non-prioritized traffic as shown in figure 10.

In I-XLP, the average packet delivery ratio is the number of packets received to the number of packets sent over all the sensor nodes. Finally, in figure 12 and figure 13 the simulation preformation of our proposed protocol studied under the data delivery with traffic differentiation based. We compare the delivery ratio of our protocol with other three layered protocols in term of both traffics. In terms of both prioritized and nonprioritized data traffic, the of average packet delivery ratio of our protocol performance shows higher delivery ratio than GPSR + S-MAC+ ESRT and GPSR + TMAC+ ESRT due to the use of link scheduling and GPSR + S-MAC+ ESRT outperform in prioritized traffic. The Flooding+CSMA outperforms average packet delivery ratio compare other protocols. 


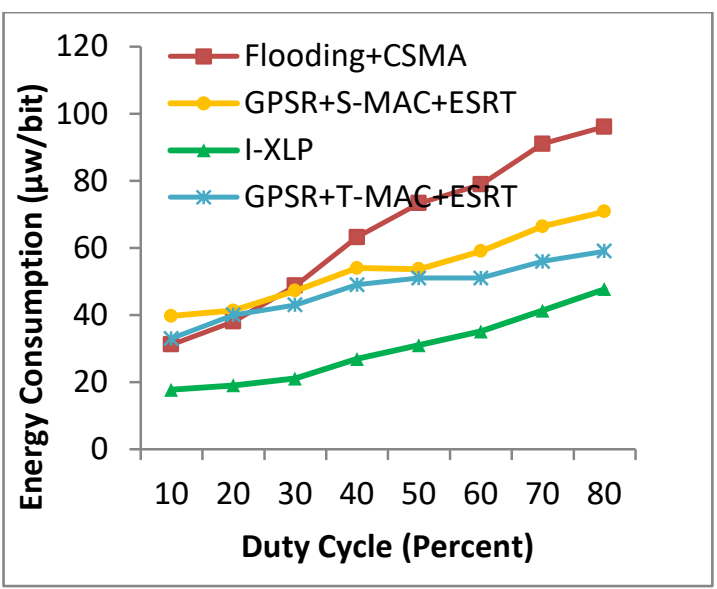

Fig. 8 Average energy consumption per bit vs. duty cycle (for non-prioritized traffic).]

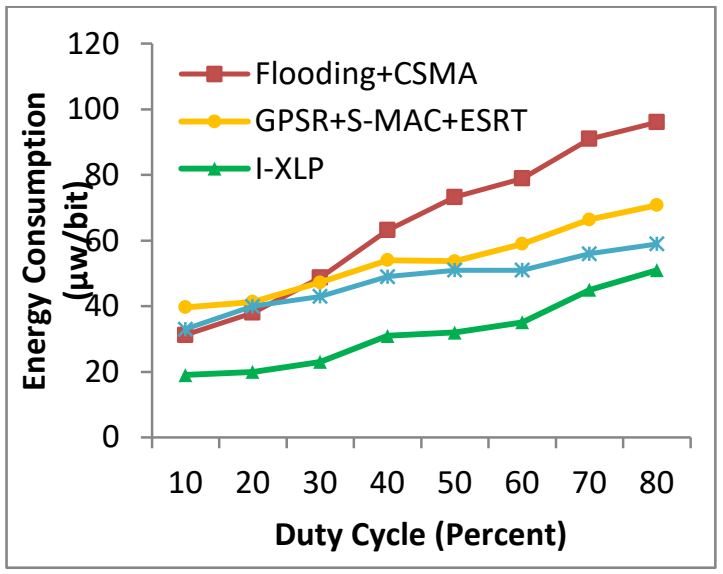

Fig. 9 Average energy consumption per bit vs. duty cycle (for prioritized traffic).

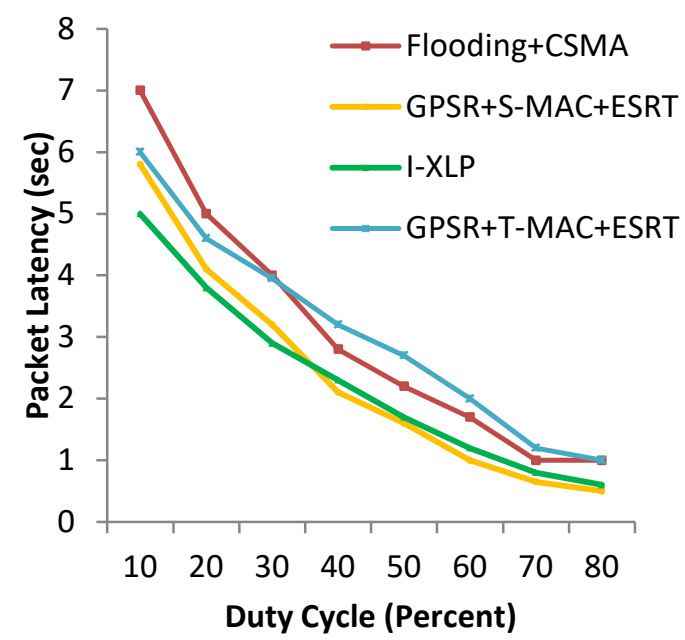

Fig. 10 Average latency vs. duty cycle (for non- prioritized traffic).

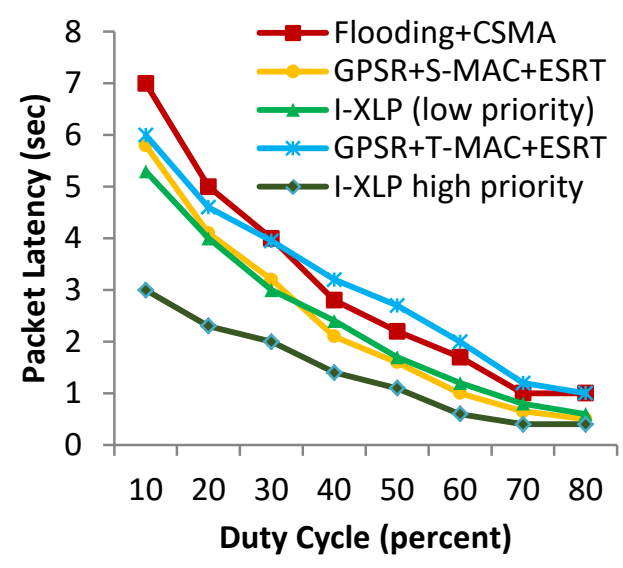

Fig. 11 Average latency vs. duty cycle (for prioritized traffic).

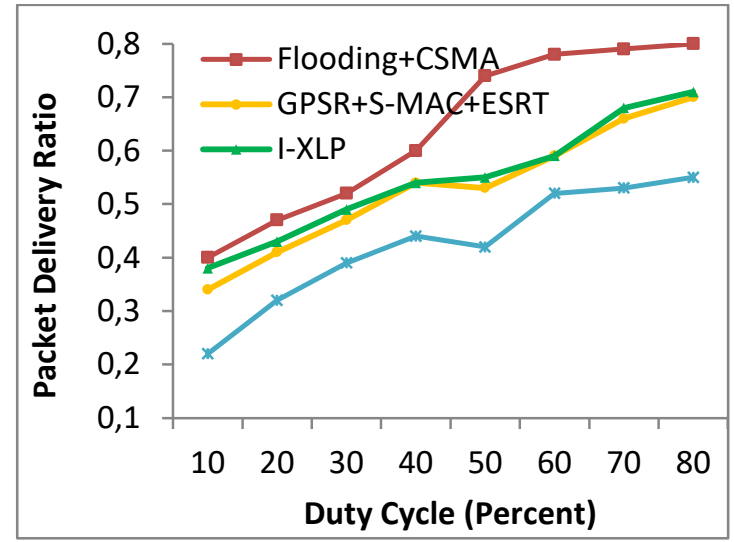

Fig. 12 Delivery ratio vs. duty cycle (for non prioritized traffic).

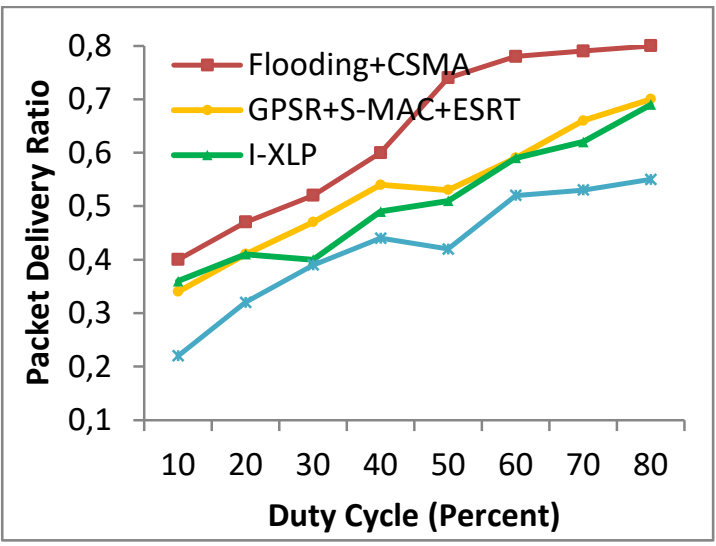

Fig. 13 Average delivery ratio vs. duty cycle (For prioritized traffic). 


\section{Conclusion}

As the sensor node and network life time efficiency are challenging issues in WSNs. I-XLP, a novel energy efficient communication QoS-aware protocol with traffic differentiation based for WSNs hase been proposed to resolve the energy consumption issues by using the strong functionalities of different layered.

Our proposed protocol, combine the functionalities of MAC, Routing and congestion control by using the concept of gradient in routing, duty cycling in the medium access control, and congestion control by pruning redundant data and all these are done in a crosslayered fashion in order to achieve optimum efficiency. Our work uses the strength of link and broadcast scheduling together in order to adjust the power adjustment feature and during data transmission our protocol achieves significant amount of energy savings as well. I-XLP protocol ensures lower latency for the prioritized traffic. We combined some other protocols to evaluate and compare the performance of our protocol with them. The simulation results show the performance improvements in respect of energy consumption efficiency and latency of our proposed protocol compared to the other protocols. As a future work, there is a scope to implement I-XLP power adjustment features, throughput, fairness and energy consumption analytical model of our proposed protocol. Besides, based on the viable energy efficient design choices presented in this article, energy efficient protocol can be designed for other application spaces of WSNs.

\section{References}

1. T. Watteyne, A. Molinaro, M. G. Richichi, and M. Dohler, "From manet to ietf roll standardization: A paradigm shift in wsn routing protocols," Commun. Surv. Tutorials, IEEE, vol. 13, no. 4, pp. 688-707, 2011.

2. M. Arifuzzaman, M. Matsumoto, and T. Sato, "An intelligent hybrid MAC with traffic-differentiation-based QoS for wireless sensor networks," Sensors Journal, IEEE, vol. 13, no. 6, pp. 2391-2399, 2013.

3. W. Ye, J. Heidemann, and D. Estrin, "Medium access control with coordinated adaptive sleeping for wireless sensor networks," Networking, IEEE/ACM Trans., vol. 12, no. 3, pp. 493-506, 2004.

4. T. Van Dam and K. Langendoen, "An adaptive energyefficient MAC protocol for wireless sensor networks," in Proceedings of the 1 st international conference on Embedded networked sensor systems, 2003, pp. 171-180.

5. W. R. Heinzelman, A. Chandrakasan, and H. Balakrishnan, "Energy-efficient communication protocol for wireless microsensor networks," in System sciences, 2000. Proceedings of the 33rd annual Hawaii international conference on, 2000, p. 10-pp.
6. L. Cheng, J. Niu, J. Cao, S. K. Das, and Y. Gu, "Qos aware geographic opportunistic routing in wireless sensor networks," Parallel Distrib. Syst. IEEE Trans., vol. 25, no. 7, pp. 1864-1875, 2014.

7. D. Ferrara, L. Galluccio, A. Leonardi, G. Morabito, and S. Palazzo, "MACRO: an integrated MAC/routing protocol for geographic forwarding in wireless sensor networks," in INFOCOM 2005. 24th Annual Joint Conference of the IEEE Computer and Communications Societies. Proceedings IEEE, 2005, vol. 3, pp. 1770 1781.

8. W. Su and T. L. Lim, "Cross-layer design and optimisation for wireless sensor networks," Int. J. Sens. Networks, vol. 6, no. 1, pp. 3-12, 2009.

9. P. Park, C. Fischione, A. Bonivento, K. H. Johansson, and A. Sangiovanni-Vincent, "Breath: an adaptive protocol for industrial control applications using wireless sensor networks," Mob. Comput. IEEE Trans., vol. 10, no. 6, pp. 821-838, 2011.

10. M. C. Vuran and I. F. Akyildiz, "XLP: A cross-layer protocol for efficient communication in wireless sensor networks," Mob. Comput. IEEE Trans., vol. 9, no. 11, pp. 1578-1591, 2010.

11. T. Kim, D. J. Love, M. Skoglund, and Z.-Y. Jin, "An Approach to Sensor Network Throughput Enhancement by PHY-Aided MAC," Wirel. Commun. IEEE Trans., vol. 14, no. 2, pp. 670-684, 2015.

12. Z. Lin and M. der Schaar, "Autonomic and distributed joint routing and power control for delay-sensitive applications in multi-hop wireless networks," Wirel. Commun. IEEE Trans., vol. 10, no. 1, pp. 102-113, 2011.

13. P. Pinto, A. Pinto, and M. Ricardo, "Cross-Layer Admission Control to Enhance the Support of Real-Time Applications in WSN," Sensors Journal, IEEE, vol. 15, no. 12, pp. 6945-6953, 2015.

14. M. Al-Jemeli and F. A. Hussin, "An energy efficient cross-layer network operation model for IEEE 802.15. 4based mobile wireless sensor networks," Sensors Journal, IEEE, vol. 15, no. 2, pp. 684-692, 2015.

15. M. Arifuzzaman and M. Matsumoto, "An Efficient Medium Access Control Protocol with Parallel Transmission for Wireless Sensor Networks," J. Sens. Actuator Networks, vol. 1, no. 2, pp. 111-122, 2012.

16. M. Arifuzzaman, M. S. Alam, and M. Matsumoto, "A hybrid MAC with intelligent sleep scheduling for wireless sensor networks," in Kaleidoscope 2011: The Fully Networked Human?-Innovations for Future Networks and Services (K-2011), Proceedings of ITU, 2011, pp. 1-7.

17. B. Karp and H.-T. Kung, "GPSR: Greedy perimeter stateless routing for wireless networks," in Proceedings of the 6th annual international conference on Mobile computing and networking, 2000, pp. 243-254.

18. Y. Sankarasubramaniam, Ö. B. Akan, and I. F. Akyildiz, "ESRT: event-to-sink reliable transport in wireless sensor networks," in Proceedings of the 4th ACM international symposium on Mobile ad hoc networking \& computing, 2003, pp. 177-188. 
19. A. Muqattash and M. Krunz, "A single-channel solution for transmission power control in wireless ad hoc network," in Proceedings of the 5th ACM international symposium on Mobile ad hoc networking \& computing, 2004, pp. 210-221.

20. J. A. Haqbeen, T.Ito, M.Arifuzzaman and T.Otsuka, "Intelligent cross layer protool with traffic diffenrtiation based QoS for WSNs," in Proceedings of the IEEE Region 10 Conference (TENCON), Singapore, 2016, pp. 1088-1092.

21. Q.Ni, L.Romdhani and T.Turletti, "A survey of QoS Enhancements for IEEE 802.11 wireless LAN," J. of Wireless communication \& mobile computing, Wiley. vol. 4, no. 5, pp. 547-566, 2004.

22. Abraham, Meethu and S.S.Singh, "A Survey on Energy peformance Improvement with Data Aggregation Techniques for WSN." Internation Journal of Computer Appications, vol. 115, no. 1, 2015.

23. K.S Yildirim and A.kantarci, "Time Synchronization Based on Slow-Flooding in Wireless Sensor Networks." IEEE Transections on Parallel and Districuted Systems, vol. 25 , no. 1,2014
24. C. Tunca, M.Y Do nnmez, S. Isık, and C. Ersoy. "An energy-efficient routing protocol for wireless sensor networks with a mobile sink". In proceeding of Signal Processing and Communications Applications Conference (SIU), 2012

25. Yu J., Qi Y., Wang G., Guo Q and Gu X. "An energy aware distributed unequal clustering protocol for wireless sensor networks". Int. J. Dister. Sensor Networks, 2011.

26. N.Vlajic and D.Xia "Wireless sensor networks: To cluster or not to cluster?". In proceeding of the 2006 International Symposium on a world of wireless, mobile and multimedia networks, 2006.

27. S.Jabbar, A.A. Manhas, M. Imran, S. Khalid and K Saleem "Energy effeicient strategy for throughput improvement in wireless sensor networks". Sensors, MDPI, vol. 15, no. 2, pp. 2473-2495, 2015.

29. I. F. Akyildiz, W.Su, Y. Sankarasubramaniam and E.Cayirci "A survey on sensor networks". IEEE Communications Magazine, vol.40, no. 8, pp. 102-144, 2002.

30. V.Srivastava and M.Motani "A survey and the road ahead". IEEE Communications Magazine, vol.43, no. 12, pp. 112-1119, 2005. 